Volume 8. No. 7, July 2020

International Journal of Emerging Trends in Engineering Research

Available Online at http://www.warse.org/IJETER/static/pdf/file/ijeter107872020.pdf

https://doi.org/10.30534/ijeter/2020/107872020

\title{
Golden Ratio and Its Application to Bayes Classifier Based Face Sketch Gender Classification and Recognition
}

\author{
Khalid Ounachad $^{1}$, Mohamed Oualla ${ }^{2}$, Abdelalim Sadiq ${ }^{3}$ \\ ${ }^{1,3}$ Department of Informatics, Faculty of sciences, Ibn Tofail University, Kenitra, Morocco \\ khalid.ounachad@uit.ac.ma a.sadiq@uit.ac.ma \\ ${ }^{2}$ SEISE: Software Engineering \& Information Systems Engineering Team, Faculty of sciences \& technology, \\ Moulay Ismail University, Errachidia, Morocco, mohamedoualla76@gmail.com
}

\begin{abstract}
Machine learning is a subarea of artificial intelligence based on the idea that systems can learn from data and make decisions automatically. Bayes Theorem is widely used in machine learning. The main objective of this paper is to classify the gender of the human being based on their face sketch images by using a golden ratio features and Bayes Classifier. This paper presents a method for human face sketch gender classification and recognition. It is inspired in our other model which was pre-trained on the same task, but with sixteen features and fuzzy approach. Toward this end, just two features will be extract from the input face sketch image based on two face golden ratios. The detection stage passes by Viola and Jones algorithm. The classification task is evaluated through Bayes classifier. An experimental evaluation demonstrates the satisfactory performance of our approach on CUFS database with $80 \%$ for training, $20 \%$ for testing. The proposed machine learning algorithm will be a competitor of the proposed relative the stat of the art approaches.
\end{abstract}

Key words : CUFS Database, Facial gender recognition, Forensic sketches, Gender classification, Golden Ratio, Machine learning.

\section{INTRODUCTION}

Face Gender Recognition (FGR) system is a major area for non-verbal language in day to day life communication. FGR systems have been attracted numerous researchers since they attempt to overcome the problems and factors weakening these systems including problem of images classification, also due to its large-scale applications in face analysis, particularly face recognition [1].

Gender based separation among humans is classified into two: male and female [2]. Face Gender Classification (FGC) systems aim to automatically classify gender in a dataset of photos or sketches images (Figure.1). It based on two-dimensional images of human subjects. Currently gender classification and recognition from facial imagery has grown its importance in the computer vision field: It play a very important function in many fields likes, face recognition [1][3], forensic crime detection [4][5], facial emotion recognition [3] and psychologically affected patients [6], night surveillance [7] and Artificial Intelligence[8][9] and soon. In this paper it can be used to identify; fastly; a criminal person from his sketch for purposes of identification [10].

Humans have a natural behaviour and ability to extract, analyze, identify, and interpret informations encrypted in the face features likes gender. The automatic task of facial gender recognition is a challenging work and explicitly difficult: Human gender classification and recognition can be done in many ways. In this paper is concerned with the gender classification based on two-dimensional images of people's face sketches.

There is a large number of databases available for human sketches gender classification and recognition research, some of them are private and some are public. The CUFS [11] is most commonly used in face sketch recognition scenario[5].

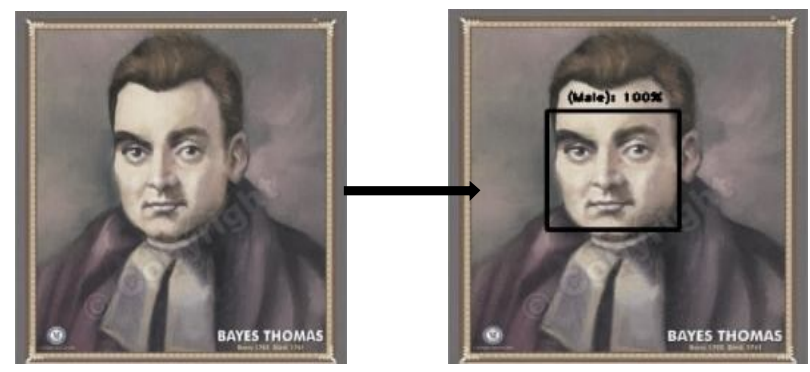

Figure 1:In the left, an input image sketch to the Facial sketch gender classification system. In the right, the output result. It indicates the detected gender with accuracy. (image is a Portrait purportedly of Bayes Thomas Bayes who is known for formulating Bayes' theorem. (images @Victory Graphik)) 
Machine learning is a subarea of artificial intelligence based on the idea that systems can learn from data and make decisions automatically. Bayes Theorem is widely used in machine learning [12], including its use in a probability framework for fitting a model to a training dataset, referred to as maximum a posteriori (MAP). We can use probability to make predictions and also in step of developing models for classification predictive modeling problems such as the Bayes Optimal Classifier and Naive Bayes. The classifier relies on supervised learning for being trained for classification. It can be trained by determining the average vector and the covariance matrices of the discriminant functions for the abnormal and normal classes from the training data. In this paper, we used Bayes classifier to solve the problem of recognizing and classifying human gender of face sketches between two different classes: male and female's face sketches subjects. The Naive Bayes classifier is a classification algorithm based on the concept of Bayes Theorem; it is a simple classifier that is based on the Bayes rules [13][14]. Bayes optimal classifier is often used to tune and harmony the discriminant function parameters of a given well-performing model on a validation database. Generally, there are three useful types of Naive Bayes model [15]: Gaussian, Multinomial and Bernoulli models. Gaussian model, assumes that features follow a normal distribution. Multinomial model, used for discrete counts and Bernoulli model. The binomial model is useful in binary feature vectors case.

Given a random rectangle whose ratio of length to width is equal approximatively to 1.618 , throughout history, this ratio has been considered the most extremely pleasing to the eye for eveone[16].This ratio was named the golden ratio by the Greeks, it is also named Phi, golden mean, golden section, extreme ratio, medial section, divine proportion, divine section, golden proportion, golden cut, golden number, and mean of Phidias[16] relatively to its mathematically, geometrically, arithmetically meaning and context use. These are three known numbers, $\Phi=1.6180339 \ldots, \varphi=0.6180339$ $\ldots$ and $\Phi 2[17], \Phi$ and $\varphi$ often called major and minor "golden section/ratio". Golden ratio was used in art, architecture, metallic glass [18] and soon.

The objective of this paper is to propose our approach based on golden ratio relationships facial and Bayes classifier to classify and recognize the gender of the input request face sketch image. This paper presents a novel method for human face sketches classification. The principle and the core of the approach include two basic aspects. The first one grants to extract two face ratios for the input face sketch and the second one allows to apply Bayes classifier by using the Gaussian model. The face golden ratios according to scientists are used to compute two golden ratios: with face and length face ratios. With ratio is the proportion between distance from pupil to pupil and the face with, from inside edge of ear to inside edge of ear. Height ratio is the proportion between distance from midpoint of eyes to mouth midpoint.
The remainder of this paper is organized as follows. Section 2 related works. Section 3 background information about golden rations, face ratios, naïve and optimal Bayes classifiers, their definitions, expressions and algorithms. In section 4, the proposed framework architecture of our approach is explained in depth. In section 5 the used Dataset and Experimental results. A conclusion discussion and future work are presented in section6.

\section{LITERATURE REVIEW}

There are several methods we had studied in the gender recognition of literature. Over the last year until Mai 2020, there has been a wealth of research in Human gender classification and recognition based on their facial images. Specially in last two years (from 2018 to March 2020) many techniques were used in this task, for example just to name a few, we briefly review related methods for facial gender classification and recognition: $\operatorname{In}[19]$ authors use feature fusion and parameter Optimization of dual-input convolutional neural network for Face Gender Recognition, their method is called the weighting fusion. MORPH is their experiments dataset. The authors of [20] use a machine learning based approach to recognize gender from real-life images. They have been trained various classifiers on many images from the audience benchmark with multiple train and test data splits. In [21] authors use motion data from multiple smart devices in their gender recognition approach. The collected motion data are according to three aspects: time, frequency, and wavelet domains. In [22] authors adopt a fused CNN of separated GEI to gender recognition in gait analysis. Kamarulzaman et Chi [23] use an intelligent gender recognition system for classification of gender in Malaysian demographic, emphasizing on deep learning-based gender recognition and HAAR Cascade classifier. In [24] the authors use a multi-task framework for facial attributes classification through end-to-end face parsing and deep CNN, they address three challenging problems of race, age, and gender recognition. In [25] authors use an Average Neural Face Embeddings (ANFE) method that uses facial vectors of people for gender recognition. In [2] we present a model which was pre-trained on face sketch gender classification and recognition task. The model is based on fuzzy hamming distance with geometric relationships called face ratios. We attempt to tune the same model for the same gender classification task but with just two features and we attempt to evaluate the approach to obtain a satisfied machine learning model on audience datasets.

The golden ratio has a very special place in mathematics, in [26] Wars study the relationships between golden ratio and regular hexagons, he confirmed that in mathematics the golden ratio is associated with a special asocial triangle hexagon with some specific angles. In [16] authors explain the golden ratio which interrelate Vitruvian Man and Neufert 
when modeling Human in information technology multimedia. Sentos et al [27] present and evaluate some selected statistical methods for identification of golden ratio. In [28] Bates describe the river Duddon volume's golden ratio wheel and spiraling "Orbicular" sonnets.

Bayes Classifiers have been used in a lot of machine learning for data classification, this model is able to make higher classification accuracy with less complexity. For example, such as in [29] authors apply naïve Bayes classification for disease prediction. In structured peer-to-peer systems the authors of [30] present an intelligent cooperative web caching approaches based on J48 decision tree and Naïve Bayes (NB) supervised machine learning algorithms. In [31] authors use Naïve Bayes classifier models for predicting the colon cancer. In [32] authors propose a customer behavior analysis using Naive Bayes with bagging homogeneous feature selection approach and soon.

\subsection{Contribution of this Work}

The contribution of this work can be summarized as:

-Comprehensive performance evaluation of off line based facial sketch gender classification and recognition by using two golden ratios in the face and Bayes classifiers.

-The development and evaluation of a geometric machine learning based human face sketch gender classification model with images from CUFS dataset, $80 \%$ for training and $20 \%$ for testing.

-The proposed approach can accurately classify the right kind of the gender's face sketch. It is inspired in our model [2] which was pre-trained on face sketch gender classification and recognition task with sixteen features. We tuned a method just with two golden ratios and Naïve Bayes supervised machine learning algorithms. This method achieves our goal by producing a classification rate reaches more than $74.34 \%$ for female and more than $85.38 \%$ for male especially in the CUFS dataset. It can be comparable with the stat of the art human face sketch gender Classifier algorithms.

\section{BACKROUND INFORMATION}

\subsection{Naive Bayesian classification method}

The naive Bayesian classification is a simple method to implement and which provides good results, despite the hypothesis strong used. This learning method is supervised. The method is based on Bayes' theorem which assumes probabilistic independence characteristics of a given group. We denote $X_{i}, i \in[0, n-1]$, the vector column $i$ of the data matrix Data.

We assume that each attribute $\mathrm{i}$ (column of the data matrix Data) can be modeled by a random variable denoted $X_{i}$ for $i \in$ $[0, n-1]$. The membership of a data in a group is modeled by the random variable $\mathrm{Y}$, whose values are discrete $\mathrm{y}_{0}=0, \mathrm{y}_{1}=$
1... The membership of the data $\mathrm{k}\left(\mathrm{k}^{\text {th }}\right.$ line of the Data matrix) is known and indicated in a vector called State.

Bayes' theorem determines on which a data item belongs to a group $y_{j}$ knowing its attributes $x_{i}$.

$$
\begin{aligned}
& \left(Y=y_{j} \mid X_{0}=x_{0}, X_{1}=x_{1}, \ldots X_{n-1}=x_{n-1}\right)= \\
& P\left(Y=y_{0}\right) \times P\left(X_{0}=x_{0}, X_{1}=x_{1}, \ldots X_{n-1}=x_{n-1} \mid Y=y_{0}\right)
\end{aligned}
$$

Where:

- $\mathrm{P}\left(Y=y_{i} \mid X_{0}=x_{0}, X_{1}=x_{1}, \ldots X_{n-1}=x_{n-1}\right):$ is the probability of belonging to the group yj knowing that the different random variables $\mathrm{Xi}$ respectively take the values $\mathrm{xi}$, - $P\left(Y=y_{i}\right)$ : is the probability of belonging to the group yj, - $P\left(x_{0}=x_{0}, X_{1}=x_{1}, \ldots X_{n-1}=x_{n-1} \mid Y=y_{i}\right):$ is the probability that the different random variables Xi take the values xi respectively knowing that the data belongs to the group yj,

- $\mathrm{P}\left(X_{0}=x_{0}, X_{1}=x_{1}, \ldots X_{n-1}=x_{n-1}\right)$ : is the probability that the different random variables $\mathrm{Xi}$ take the values $\mathrm{xi}$ respectively.

The naive Bayesian hypothesis assumes that all attributes are independent and therefore that:

$$
\begin{aligned}
P\left(X_{0}=\right. & \left.x_{01} X_{1}=x_{1}, \ldots X_{n-1}=x_{\mathrm{n}-1} \mid Y=y_{j}\right) \\
& =\prod_{i} P\left(X_{i}=x_{i} \mid Y=y_{j}\right)
\end{aligned}
$$

As the denominator $\mathrm{P}\left(X_{0}=x_{0}, X_{1}=x_{1}, \ldots X_{n-1}=x_{n-1}\right)$ is independent of the group considered and therefore constant, we only consider the numerator:

$P\left(Y=y_{i}\right) \times \mathrm{P}\left(X_{0}=x_{0}, X_{1}=x_{1}, \ldots, X_{n-1}=x_{n-1}\right)$

To determine the most likely group to which a datum $\mathrm{z}$ to be classified belongs, represented by the tuple $\left(\Sigma_{0}, \Sigma_{1}, \ldots, \Xi_{n-1}\right)$, we choose the maximum probability:

$P\left(Y=y_{i}\right)_{X} \Pi_{i} P\left(X_{i}=z_{i} \mid Y=y_{i}\right)$

Among the $\mathrm{j}$ groups. Various probability laws are used to estimate $P\left(X_{i}=z_{i} \mid Y=y_{j}\right)$, we use a Gaussian distribution law.

To calculate the conditional probability $P\left(x_{i}=z_{i} \mid Y=y_{i}\right)$ of a datum $\mathrm{z}$ represented by the tuple, we use a Gaussian distribution of the form:

$P\left(X_{i}=z_{i} \mid Y=y_{j}\right)=\frac{1}{\sqrt[2]{2 \pi \sigma^{2}}} e^{-\frac{\left(x_{i}-\mu_{\left.x_{i} y_{j}\right)^{2}}\right.}{2 \sigma^{2} x_{i} y_{j}}}$

where $\mu_{x_{i} y_{i} \text { and }} \mu^{2}{ }_{x_{i} y_{i}}$ are the mean and variance of the random variable $\mathrm{Xi}$, estimated from the values attribute data 
from the i-th column of the vector Data for the group corresponding to $\mathrm{y}_{\mathrm{j}}$.

We recall that, for a $\mathrm{n}$ dimension vector $\mathrm{x}$ :

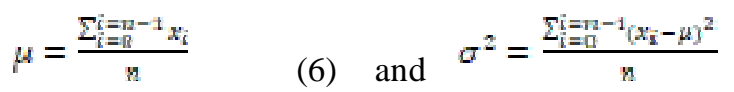

\subsection{Golden Ratio}

The irrational number $(\sqrt{ } 5-1) / 2$ is known as phi denoted by $\Phi / \varphi$, defined by Euclid of Alexandra [27]. $\Phi=$ $1.6180339 \ldots, \varphi=0.6180339$.

The with face ratio W_FR is the proportion between distance from pupil to pupil and the face with, from inside edge of ear to inside edge of ear. The Height face ratio H_FR is the proportion between distance from midpoint of eyes to mouth midpoint. (Figure.2). The shape of the face was perfect or golden when its W_FR was equal to 0.46 and H_FR was equal to 0.36 (Figure.3).

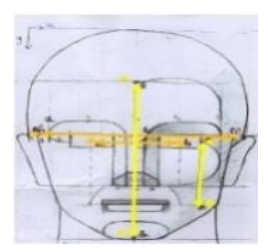

Figure 2: Our face sketch ratio model. It shows the different selecting distances to calculate $W_{F B}$ and $\boldsymbol{H}_{F R}$.

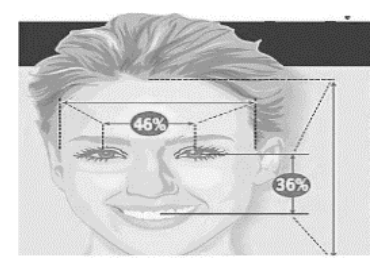

Figure 3: Jessica Alba face. She has a face that meets the golden ratio according to scientists and she has the most average face proportions. That average was beautiful. \{image: Daily Mirror

\section{RESEARCH METHODOLOGY}

An overview of our proposed research methodology framework based on Bayes Classifier with two face ratios for face sketch gender classification is shown in Figure 4. In detail, our system has two modes, in both them, the input facial sketch image and all face sketches of the dataset are converted to a Gray level, they are resized and cropped into 200x250 pixels. These dimensions are chosen: It's the proposed default choice of the datasets used and it is also the dimensions used in our related work. Facial images detection passes though viola johns facial detection algorithm. In the offline phase, the first step of the system is to normalize and to pretrain all sketches. For that they have been transformed into Gray level images and they are all cropped to 200x250 pixels. The same technique steps are thus used to the online mode. After this stage, to detect the faces of the images, we projected the viola and jones algorithm. The result that follows this second step is used to locate the 68_point_landmarks in each face. These 68 points will be the parameter of a geometric descriptor which allows to extract an identity of each face via the calculation of ratios $W_{F R}$ and $H_{F R}$. A vector will be dedicated to group these harmonious and beautiful distances in order. This vector represents a real proportionality with any other similar vector. The ratios of these distances in the vector have been stored as already detailed in last section 3.2. In online process of the face sketch gender classification system, given a facial sketch image, two features are extracted based on four distance as showed in Figure. 2 and Figure. 3 and defined in last section. These characteristics composes a vector of real values. This vector is considered as an identifier of the face image from which the values have been extracted and calculated. In offline process of the facial sketch gender classification System, the same distances used in online process are extracted and calculated for each facial sketch image request. The face ratios for each input facial sketch image is then used as feature vectors for final classification and recognition system based on Bayes Classifier.

The Bayes classifier algorithm contained five steps : Dataset separation by gender classes. Summarization Dataset. Summarization Data by classes. Gaussian probability density function definition and class probabilities calculation. 


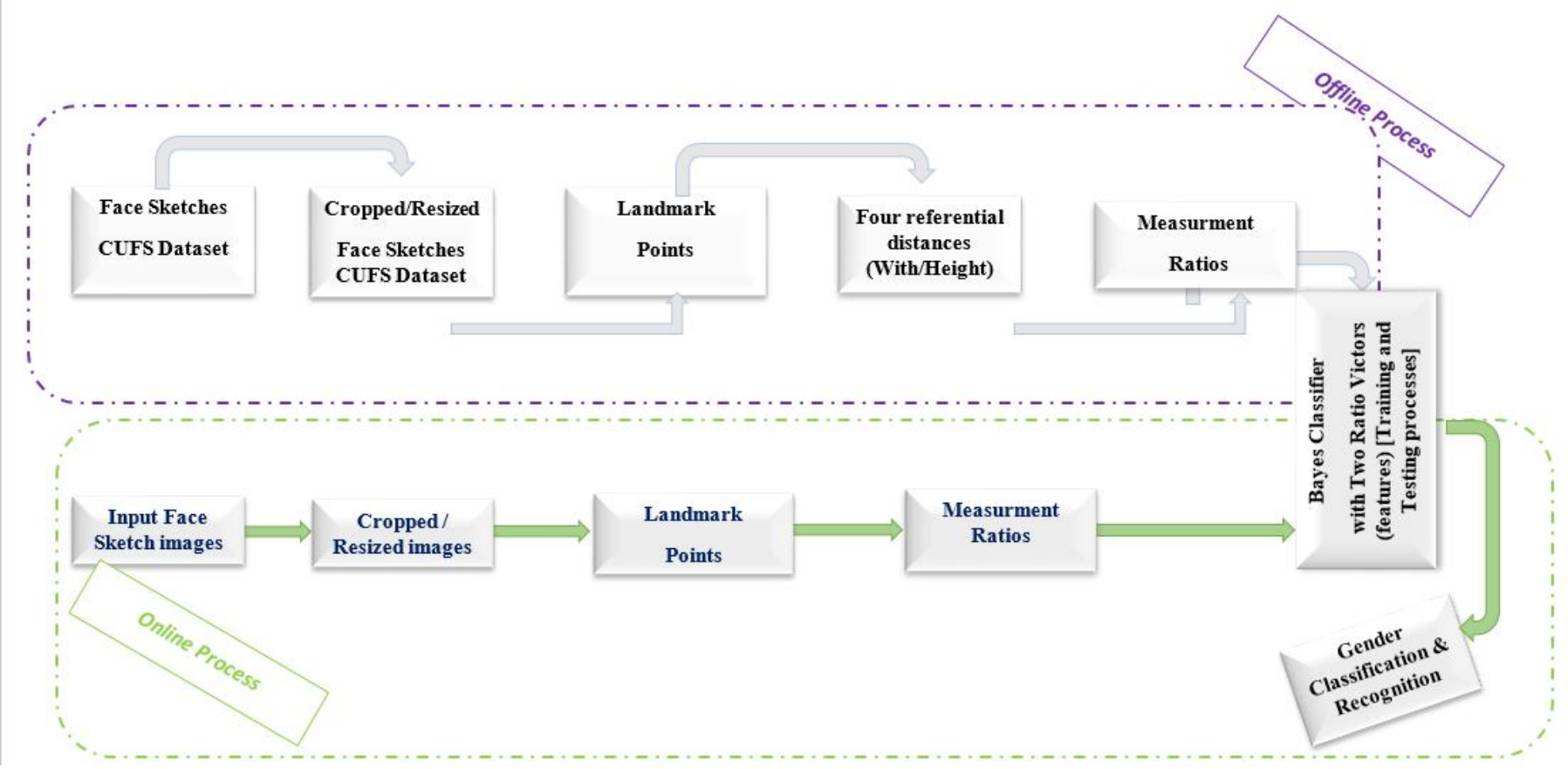

Figure 4:An overview of our proposed research methodology framework based on Bayes Classifier with two face ratios for Face sketch Gender Classification (FSGC)

\section{EXPERIMENTS RESULTS AND DISCUSSION}

There is a large number of databases available for human face sketch classification and recognition research, some of them are public and some are private. The CUFS database [2][5][8] is public and most commonly used in face sketch recognition research. CUHK Face Sketch database (CUFS) is for research on face sketch synthesis and face sketch recognition. It includes 188 faces from the Chinese University of Hong Kong (CUHK) student database, 123 faces from the AR database and 295 faces from the XM2VTS database. There are 606 faces in total. For each face, there is a sketch drawn by an artist based on a photo taken in a frontal pose, under normal lighting condition, and with a neutral expression. In Table 1 CUFS gender statistics is detailed whilst the Figure. 5 the gender distribution.

Table 1: The Gender statistics from CUFS Dataset

\begin{tabular}{|c|c|c|c|}
\hline $\begin{array}{c}\text { Database } \\
\text { name }\end{array}$ & $\begin{array}{c}\text { Male } \\
\text { Images }\end{array}$ & $\begin{array}{c}\text { Female } \\
\text { Images }\end{array}$ & $\begin{array}{c}\text { Total } \\
\text { images }\end{array}$ \\
\hline CUHK & 133 & 54 & 188 \\
\hline AR & 74 & 49 & 123 \\
\hline XM2VTS & 179 & 116 & 295 \\
\hline
\end{tabular}

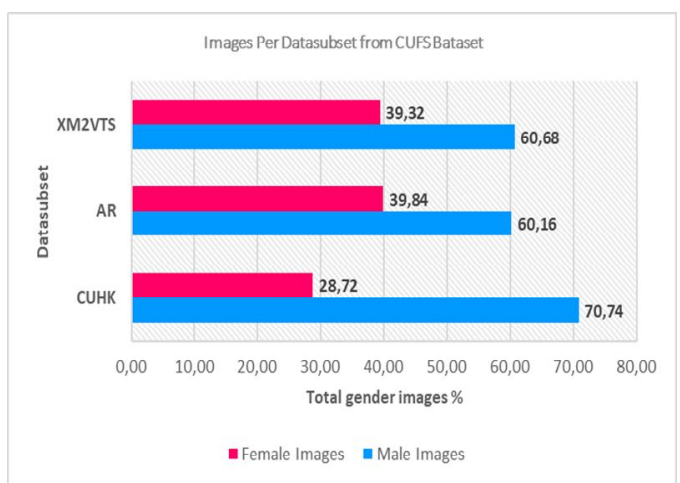

Figure 5:Our used CUFS gender distribution

To demonstrate the e $\square$ ectiveness of the proposed method, we proceeded for acquiring the CUFS Dataset. We used $80 \%$ of database images for training and $20 \%$ for testing. Figure 8 clearly illustrates step by step the results obtained as we progress in the process of the framework already described previously. in the first step the extract face sketches of the dataset. In the second: The cropped Face sketches. In the third: The extract 68 face landmarks points. In the fourth: calculate of the two features and in the last step: the ratios vector generated from the facial images, it is involved to classify the kind of the facial gender of the input facial sketch. The output result of Face Sketch Gender Classification System (FSGCS) is the probe kind of gender. TABLE 2 shows the Accuracy per gender for our approach based on Bayes classifier and two golden face ratios. It reports the facial sketch gender classification and recognition accuracies using a supervised machine algorithm based on probability. We 
calculate two posteriors using gaussian naive Bayes classifier, it is probably the most popular type of Bayes:

$$
\begin{aligned}
& \text { posterior (male })=\frac{\mathrm{P}(\text { male }) \mathrm{p}\left(H_{F R} \mid \text { male }\right) \mathrm{p}\left(W_{F R} \| \text { male }\right)}{\text { marginal probability }} \\
& \text { posterior (female })=\frac{\mathrm{P}(\text { female }) \mathrm{p}\left(H_{F R} \mid \text { female }\right) \mathrm{p}\left(W_{F R} \| \text { fenale }\right)}{\text { manginal probability }}
\end{aligned}
$$

Table 2: The Gender statistics from CUFS Dataset

\begin{tabular}{|c|c|c|}
\hline Gender & Female & Male \\
\hline Accuracy $\%$ & $74.34 \%$ & $85.38 \%$ \\
\hline
\end{tabular}

Figures 6 shows comparison of cumulative match scores in our facial images gender classification method using Bayes classifier. It measures the percentage of the probe gender. The $\mathrm{x}$-axis represents the gender and the y-axis represents the classification rate. The results clearly demonstrate the superiority of our algorithm to more classify male, but the last rate of classification is that of the female gender. The result tests deduct that the estimate rate reaches more than $74.34 \%$ for male and more than $85.38 \%$ for female. In Figure.7. a comparison between our method and our last face sketch gender recognition other approach [2]. Two approaches help to better recognize the male gender but not able the recognize the female gender. This accuracy inequality is due to some parameters analyzed in [1] where the authors present a comprehensive analysis of how and why face recognition accuracy differs between men and women. We recall that in face sketch gender recognition approach based on Bayes classifier we use just two golden face ratio features, however the second approach based on FHD (Fuzzy Hamming
Distance) and averages method [2] we use sixteen features and the result accuracy is almost the same approximately.

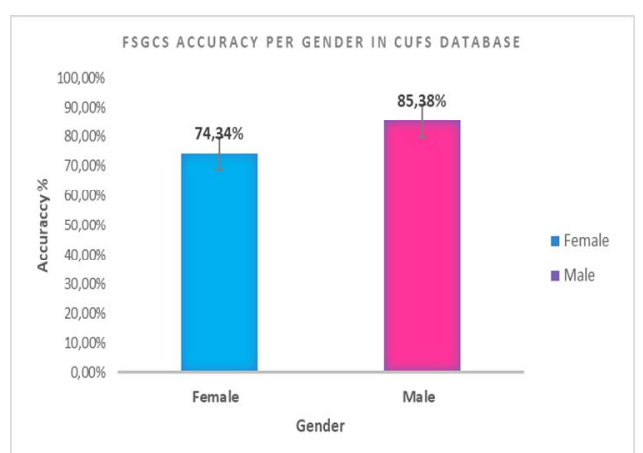

Figure 6: Performance of Golden ratio and Bayes Classifier approach vs Gender (CUFS Database)

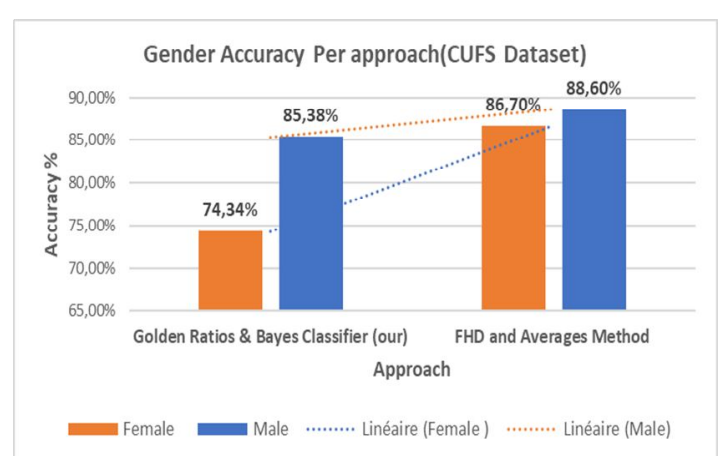

Figure 7: Comparison between our machine learning approaches for FSGCS

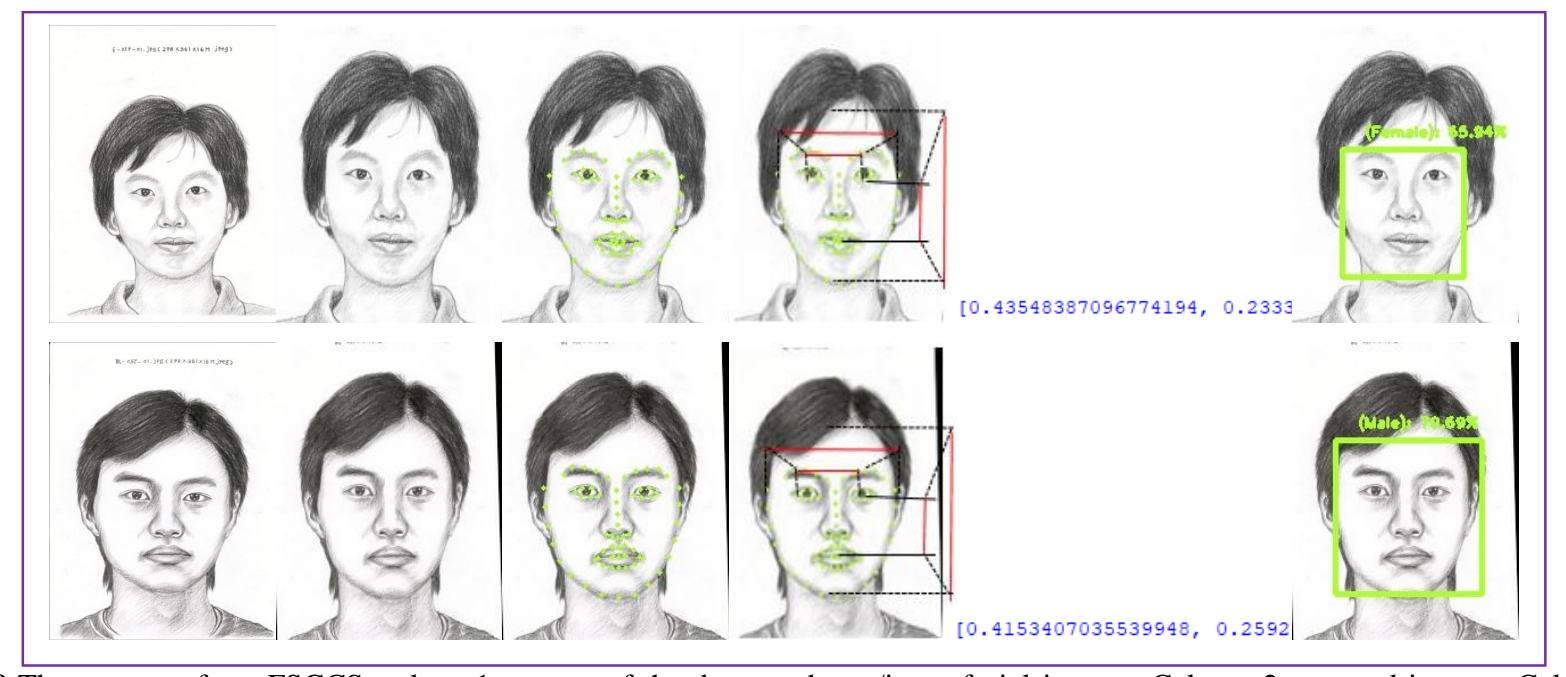

Figure 8: The process of our FSGCS, column1: extract of the dataset photos/input facial images. Column 2: cropped images. Column 3: extract the 68 face landmarks points. Column 4: calculate of two features and Column 5: the ratio vector generated from the facial sketches; it is involved to estimate the kind of gender. The output result of FSGCS is the probe kind of gender of the input facial images. 
To use our algorithm perfectly:

-Large and labelled face sketch dataset is needed with facial images useful for gender classification.

-Facial frontal and net images are needed, Figure.

-A structured and multiple computing power are required for training and testing stages.

-Large memory, powerful operating system and efficient platform are demanded.

\section{CONCLUSION}

This paper proposes a new geometrical approach for facial gender recognition. The method is based on Bayes classifier and two golden Face Ratios. We used two features based on the with face ratio and $W_{F R}$ the Height face ratio $H_{F R}$. We tested our method on CUFS dataset and the results is very satisfactory. Our work is inspired by the recent successful methods that showed that relatively simple geometrics features could be used to give good performance in machine learning algorithms based framework. Our approach Can be useful in the process of identification of the criminal and the future work will include a comparison of our algorithms with other algorithms on the domain, it will include also an automatic decrease in the number of the features used in our last face sketch gender recognition other approach[2].

\section{REFERENCES}

[1] V.Albiero, K.S.Krishnapriya, K.Vangara, K.Zhang, M.C. King and K.W. Bowyer. Analysis of Gender Inequality In Face Recognition Accuracy. The IEEE Winter Conference on Applications of Computer Vision (WACV) Workshops, 2020, pp. 81-89. January 2020.

[2] K.Ounachad, A. Souhar and A. Sadiq. Face Sketch Recognition: Gender Classification and Recognition. International Journal of Psychosocial Rehabilitation .Vol.24(03),pp. 1073-1085. Febreary 2020. https://doi.org/10.37200/IJPR/V24I3/PR200860

[3] K.Ounachad, M. Oualla, A. Souhar and A. Sadiq. Geometric Feature Based Facial Emotion Recognition. International Journal of Advanced Trends in Computer Science and Engineering. Vol.9. pp. 3417-3425.May- June 2020.

[4] M.Jacquet, and C.Champod. Automated face recognition in forensic science: Review and perspectives. Forensic Science International. 2020 Feb, vol.307:110124. Febreary 2020. https://doi.org/10.1016/j.forsciint.2019.110124

[5] K.Ounachad,M. Oualla, A. Souhar and A. Sadiq. Face Sketch Recognition-An Overview. Proceedings of the 3rd International Conference on Networking, Information Systems \& SecurityArticle No.: 38 . pp. 1-8. March 2020
[6] G.Simcock, L.T.McLoughlin, T.D.Regt, K. M.Broadhouse, D.Beaudequin, J. Lagopoulos and D.F.Hermens. Associations between Facial Emotion Recognition and Mental Health in Early Adolescence. sds Int J Environ Res Public Health. 2020 Jan, vol.17(1): 330. January 2020. https://doi.org/10.3390/ijerph17010330

[7] Z.Kadim, M.A. Zulkifley and N.Hamzah. Deep-learning based single object tracker for night surveillance. International Journal of Electrical and Computer Engineering (IJECE), vol. 10(4), pp. 3576-3587. August 2020.

[8] K.Deepa, R.K. LekhaSree, B. Renuga Devi, V.Sadhana and S.Virgin Jenifer. Cervical Cancer Classification. International Journal of Emerging Trends in Engineering Research. Vol. 8. Pp. 804-807. March 2020.

https://doi.org/10.30534/ijeter/2020/32832020

[9] Aaron Don M. Africa, Anna Rovia V. Tabalan, Mharela Angela A. Tan. Speech Emotion Recognition using Support Vector Machines. International Journal of Emerging Trends in Engineering Research. Vol. 8. Pp. 1212-1216. April 2020. https://doi.org/10.30534/ijeter/2020/43842020

[10] K.Ounachad , A. Souhar and A. Sadiq. Fuzzy Hamming distance and Perfect Face Ratios based Face Sketch Recognition. IEEE International Congress on Information Science and Technology (CiSt) Marrakech, Morocco. pp.317-322.October 2018.

[11] X. Wang and X. Tang. Face Photo-Sketch Synthesis and Recognition. IEEE Transactions on Pattern Analysis and Machine Intelligence (PAMI), vol. 31( 11), pp.1955-1967. November 2009.

[12] C.S.Chandana , K.D. Rao and P. K.Sahoo. Face Recognition through Machine Learning of Periocular region. International Journal of Engineering Research \& Technology (IJERT,vol. 91 03), pp362-365. March 2020. https://doi.org/10.17577/IJERTV9IS030387

[13] E Alpaydin. Introduction to machine learning. books.google.com. 2020 .

[14] A.Vinaya, A.Guptaa, A.Bharadwaja, A.Srinivasana, K.N.B.Murthya and S.Natarajana. Unconstrained Face Recognition using Bayesian Classification. Procedia Computer Science,vol.143, , $p p$. 519-527.2018.

[15] K.MKaarthik, J.Madhumitha , T.Narmatha and S.S.Barani. Face Detection and Recognition using Naïve Bayes Algorithm. International Journal of Disaster Recovery and Business Continuity, vol.11, No. 1,pp.11-18. March 2020.

[16] E. Abu-Taieh, M. El-Maheed, M.El-Maheed, A. Abu-Tayeh, J. Abu Tayeh, A.El-Haj. Human Modeling in Information Technology Multimedia Using Human Biometrics Found in Golden Ratio Vitruvian Man and Neufert. Innovation Vision 2020: Sustainable 
growth, Entrepreneurship, and Economic Development.pp.858-869. March 2020.

[17] S. P. Klykov. S-shaped growth curves in fermentations and golden ratio. International Journal of Biomathematics, vol.13(3). March 2020.

[18] Y.Q. Jiang and P. Peng. Nearly golden-ratio order in Ta metallic glass. Chinese Physics B, vol.29(4).March 2020

[19] C.J. Lin , C.H.Lin and S.Y.Jeng. Using Feature Fusion and Parameter Optimization of Dual-input Convolutional Neural Network for Face Gender Recognition. Applied Sciences (Advances of Computer Vision special issue), vol.10(9). April 2020.

[20] A. Balyan, S. Suman, N. Z. Naqvi and K.Ahlawat. Gender Recognition from Real-Life Images. In: Solanki V., Hoang M., Lu Z., Pattnaik P. (eds) Intelligent Computing in Engineering. Advances in Intelligent Systems and Computing, Springer, vol.1125,pp. 127-134. 2020.

[21] J.Dong, Y.Du and Z.Cai. Gender recognition using motion data from multiple smart devices. Expert Systems with Applications, vol.147. June 2020. https://doi.org/10.1016/j.eswa.2020.113195

[22] J.Deng, S.Bei, S.Shaojing, T.Xiaopeng and Z.Zhen. Gender Recognition via Fused CNN of Separated GEI. IEEE 4th Information Technology, Networking, Electronic and Automation Control Conference (ITNEC). May 2020.

[23] Y.S.Chi and S.F. Kamarulzaman. Intelligent Gender Recognition System for Classification of Gender in Malaysian Demographic. In: Kasruddin Nasir A. et al. (eds) InECCE2019. Lecture Notes in Electrical Engineering, vol 632. Springer, Singapore.pp. pp 283-295. 2020

[24] K. Khan, M. Attique, R.U. Khan, I. Syed and T.S. Chung. A Multi-Task Framework for Facial Attributes Classification through End-to-End Face Parsing and Deep Convolutional Neural Networks. Sensors (Image and Video Processing and Recognition Based on Artificial Intelligence), vol. 20(2), 328. January 2020

[25] S.Makinist, B.Ay and G.Aydin. Average Neural Face Embeddings for Gender Recognition. Avrupa Bilim ve Teknoloji Dergisi, Year 2020, Volume, pp.522 - 527. April 2020. https://doi.org/10.31590/ejosat.araconf67

[26] A.Wares. The golden ratio and regular hexagons. International Journal of Mathematical Education in Science and Technology, vol.51,pp.157-160. March 2020.

[27] M.M.G.Santos, L.A.Beijo, F.Goecking and A.J.Petrini. Statistical methods for identification of golden ratio. Biosystems, vol.189. Marh 2020.

[28] B. Bates. The River Duddon Volume's Golden Ratio Wheel and Spiraling "Orbicular" Sonnets. The Wordsworth Circle, vol.51. Winter 2020.
[29] M.Sharad, J.Bhavesh. Application of naïve bayes classification for disease prediction. nternational Journal of Management, IT and Engineering, vol.9, pp.80-87.April 2019.

[30] I.Hamidah. Intelligent cooperative web caching policies for media objects based on J48 decision tree and Naïve Bayes supervised machine learning algorithms in structured peer-to-peer systems. Journal of Information and Communication Technology, vol. 15, pp. 85-116. January 2020.

[31] N.Salmi and Z.Rustam. Naïve Bayes Classifier Models for Predicting the Colon Cancer. IOP Conf. Series: Materials Science and Engineering 546. July 2019.

[32] R.S.Subramanian and D. Prabha. Customer behavior analysis using Naive Bayes with bagging homogeneous feature selection approach. Journal of Ambient Intelligence and Humanized Computing . April 2020

https://doi.org/10.1007/s12652-020-01961-9 